\title{
Fenton Reaction Facilitates The Fungal Infection On The Unicellular Alga Haematococcus Pluvialis and Discovery of a Biosafe and Environment-Friendly Chemical That Blocks The Infection
}

\section{Hailong Yan}

Institute of Hydrobiology Chinese Academy of Sciences

\section{Haiyan Ma}

Institute of Hydrobiology Chinese Academy of Sciences

Yanhua Li

Institute of Hydrobiology Chinese Academy of Sciences

Liang Zhao

Demeter Bio-Tech CO., LTD.

Juan Lin

Jiujiang University

Qikun Jia

Institute of Hydrobiology Chinese Academy of Sciences

Qiang Hu

Shenzhen University

Danxiang Han ( $\nabla$ danxianghan@ihb.ac.cn )

Institute of Hydrobiology Chinese Academy of Sciences https://orcid.org/0000-0003-4166-9299

\section{Research}

Keywords: Haematococcus pluvialis, Fungal pathogen, Secondary metabolites, Oxidative stress, antioxidant

Posted Date: December 14th, 2021

DOI: https://doi.org/10.21203/rs.3.rs-1147317/v1

License: (1) This work is licensed under a Creative Commons Attribution 4.0 International License. Read Full License 


\section{Abstract \\ Background}

The green microalga Haematococcus pluvialis is used as cell factories for producing astaxanthin, the high-value natural compound with multiple biological functions. However, H. pluvialis is prone to the infection by a parasitic fungus Paraphysoderma sedebokerense, which is the most devastating threat to the mass culture of $H$. pluvialis all over the world. Little is known for the mechanisms underlying the infection process, though it is of great essential for developing effective measures to mitigate the pathogen threatening for the natural astaxanthin industry.

\section{Results}

We observed that there were heat-stable substances with small molecular weight produced during the infection process, which significantly stimulated the parasitism process and enhanced the susceptibility of $H$. pluvialis cells to the pathogen. Systematic approaches including multi-omics, biochemical and imaging analysis were deployed to uncover the identity of the metabolites and the underlying mechanisms. Two metabolites, 3-hydroxyanthranilic acid and hordenine were identified and proved to stimulate the infection via driving fenton reaction mediated oxidative stress to $H$. pluvialis. The reaction

generated hydroxyl radicals to disrupt the subcellular components of the algal cells and to make the algal cells more susceptible to the infection. Based on these findings, a biosafe and environment-friendly antioxidant butylated hydroxyanisole was selected to inhibit the fungal infection, which completely abolished the infection at $12 \mathrm{ppm}$.

\section{Conclusions}

This study provide for the first time, a framework to dissect the functions of secondary metabolites in the interaction between the unicellular algal $H$. pluvialis and its fungi parasite, indicating that oxidative degradation is a strategy used for the fungal infest. Eliminating the oxidative burst through adding antioxidant butylated hydroxyanisole could be an effective measure to reduce parasitic infection in $H$. pluvialis mass culture.

\section{Background}

Microalgae are increasingly used for producing high-value compounds for food, nutraceuticals and cosmetics applications and are promising resources for biofuels production (Borowitzka \& Vonshak, 2017; Torres-Tiji et al., 2020). Microalgae also possess great potentials in greenhouse gas emission mitigation as well as for wastewater treatment (Choi et al., 2019; Maity et al., 2014; Specht et al., 2010). However, contaminations of parasites in mass culturing system are keeping threatening the sustainable production of biomass and bio-products by utilization of microalgae (Carney \& Lane, 2014). 
Consequently, it is difficult to produce bulk volume of microalgal biomass at low cost due to the occurrence of various predators and pathogens in mass cultivation.

The green unicellular microalga Haematococcus pluvialis is a freshwater biflagellate and belongs to the class Chlorophyceae, order Volvocales, which is well known for its ability in accumulating up to $5 \%$ of the dry weight biomass of natural bio-active compound astaxanthin under stresses conditions such as nitrogen deficiency and high light irradiation (Damiani et al., 2010; Han et al., 2013; Harker et al., 1996; Ren et al., 2021). Therefore, $H$. pluvialis is considered as the most sustainable feedstock for the commercial production of astaxanthin (Khoo et al., 2019), which has an estimated market value of USD 240 million in 2021 (Ren et al., 2021). However, the development of H. pluvialis mass culture industry has been retarded by contaminations of fungal parasite, which is often accompanied by reduced biomass yield and astaxanthin productivity (Ding et al., 2020; Shah et al., 2016). A parasitic fungus Paraphysoderma sedebokerense (Blastocladiomycota) infect the $H$. pluvialis cells in a highly speciesspecific manner, which is the most devastating threat to the mass culture of $H$. pluvialis all over the world (Ding et al., 2020; Hoffman et al., 2008; Hwang et al., 2019). Once the pathogen appears in the mass culturing system, the infected algal cells are dying during very short period of time, causing severe economic loss for the natural astaxanthin manufacturing industry (Gutman et al., 2009; Strittmatter et al., 2016). Several strategies have been developed to control the pathogenic fungi, such as maintaining the $\mathrm{pH}$ of culture system at the acidic condition and application of sodium dodecylbenzene sulfonate (SDBS) (Ding et al., 2020; Hwang et al., 2019). More environment-friendly and biosafe measures are desired and necessary to improve the sustainability of $H$. pluvialis mass culture.

Understanding about the mechanisms underlying the infection process is essential for developing effective measures to mitigate the pathogen threatening for the natural astaxanthin industry. Interactions between $H$. pluvialis and $P$. sedebokerense have been investigated in previous studies. Sugar moiety on the $H$. pluvialis cell wall was proposed to play a role as binding molecule for recognition by $P$. sedebokerense (Gutman et al., 2011). Heat-stable recognition sites on the algal cell were necessary for the fungal attachment and encystment, while signal transduction in $\mathrm{H}$. pluvialis cells was obligated for the fungal sporangium development and epidemic (Asatryan et al., 2019). Interaction between the cell walls of $H$. pluvialis and carbohydrate activated enzymes in $P$. sedebokerense was believed to be essential for the parasitism process (Lin et al., 2021). These studies are focused on the specific recognition of $H$. pluvialis cells by $P$. sedebokerense and the warfare between them during the early-stage of infection, but little is known for the mechanisms underlying the post-infection process, especially for the fast material degradation in algal cells.

In this study, we observed that the accumulated secondary metabolites in the infection system significantly stimulated the parasitism process and enhanced the susceptibility of the algal cells to the pathogen. With the aim to uncover the identity of them and to understand the underlying mechanisms, systematic approaches including multi-omics, biochemical and imaging analysis were deployed herein. Two metabolites, i.e. 3-hydroxyanthranilic acid (3-HAA) and hordenine, were identified and proven stimulating the infection process via non-enzymatic fenton reaction in a congruent manner. The hydroxyl 
radicals produced from the reaction promoted the infection through causing strong oxidative stresses to the host cells and impairing the algal cell structures along with degradation of the intracellular components. Intentionally, application of the antioxidant butylated hydroxyanisole (BHA) to the algalfungal system reduced the infection ratio effectively, indicating that oxidative degradation is a strategy used for the fungal to successfully infect algal cells while eliminating the oxidative stress was practicable for mitigating fungal infection in $\mathrm{H}$. pluvialis mass culture. This study provided a framework to dissect the functions of secondary metabolites in the interaction between the unicellular algal and its fungal pathogen, and developed a new crop protection measure to improve the sustainability of algal mass cultivation.

\section{Results And Discussion}

\section{Fungal infection caused cell death of $H$. pluvialis and the supernatant post infection (SPI) enhanced the infection process}

When the H. pluvialis cells cultivated in $360 \mathrm{~L}$ panel photobioreactors were infected by $P$. sedebokerense, algal cells died rapidly and seriously (Fig. 1A-B). We collected the supernatant post infection (SPI) by removing both the host and fungal cells via centrifugation, and investigated the effects of SPI on the newly infection process. When the healthy algal cells were pre-treated with SPI and then challenged with the fungal swarmers, the infection process was significantly promoted (Fig. 1C). On the second day post inoculation, the color of the control cell culture was dark-green and a few algal cells were attached with the fungal swarmers. By contrast, a large number of dead algal cells were observed in the culture of algal cells pre-treated with SPI, which settled down to the bottom of flask. On the third day post inoculation, the color of the culture of algal cells pre-treated with SPI turned to brownish, whereas the control remained green. Pre-treating the algal cells with SPI prior to the pathogenic challenge caused significantly higher infection ratio after the second day post inoculation than the control (Fig. 1D). Additionally, the SPI remained the infection enhancing activity after pre-treated in $98^{\circ} \mathrm{C}$ water bath for $15 \mathrm{~min}$, indicating that the activity of SPI was not eliminated by heating. It was further observed that in the 48h-SPI treated algal cells, the pigments were partially degraded and the cellular starch granules disappeared (Fig. 1E). Quantitative analysis revealed that when compared to the control, the contents of the total cellular carbohydrates and pigments (i.e. carotenoids and chlorophyll) were reduced in the algal cells treated with SPI by $50 \%$ and $20 \%$, respectively (Fig. 1F). Additionally, after filtered with 3000 Da cut-off membrane, the activity of the filtrate did not significantly altered in degrading carbohydrates and pigments in the algal cells, suggesting that the activity in the SPI was most likely attributable to small molecules. These results together suggested that some heat-stable substances with small molecular weight produced during the infection process and were capable of enhancing the susceptibility of $H$. pluvialis cells to the pathogen by affecting the algal cell integrity.

\section{SPI induced oxidative stresses within algal cells}


Alterations in the subcellular structures of the algal cells challenged with both $P$. sedebokerense and SPI were observed with transmission electron microscopy (TEM) (Fig. 2A). Degradation of the subcellular membrane systems were observed in the algal cells treated with SPI without the involvement of fungus. Additionally, the algal cell walls were loosened after being treated with SPI. These results indicated that the substances in SPI degraded the algal cellular components and destructed membranes.

To uncover the identity of the secondary metabolites in SPI, transcriptomic analysis was conducted to facilitate understanding the effects of SPI on the algal cells. A total of 998 and 490 genes were up- and down-regulated in $\mathrm{H}$. pluvialis, respectively, after the SPI treatment for $24 \mathrm{~h}$. Expression of many genes involved in biotic stresses responses were significantly altered in the algal cells treated with SPI (Fig. 2B). Several genes coding for the anti-oxidative enzymes were found to be significantly up-regulated while genes coding for synthesis and transportation were down-regulated. Up-regulation of the genes involved in oxidative stress responses indicated that SPI may contain substances that can cause the generation of ROS (Hasanuzzaman et al., 2020; Torres et al., 2006). To test this hypothesis, the oxidative activities of SPI were measured by using the thiobarbituric acid (TBA) assay with fenton reagent as the positive control because it is a known reaction that generates oxidative stress through small molecules (Arantes et al., 2012; Eastwood et al., 2011). The results showed that the SPI possessed strong oxidative activity in vitro (Fig. 2C). In addition, the SPI showed lipid peroxidation activity when acting on the algal cellular membranes, leading to formation of malondialdehyde (Fig. 2D). To further identify the ROS produced by SPI, the dimethyl sulfoxide trapping method was used and the results suggested that SPI could produce hydroxyl radical in vitro (Fig. 2E).

Based on the transcriptomic results and a suite of observations and biochemical assays, it can be concluded that SPI contained substances that exerted oxidative stresses via generation of ROS in the algal cells. Oxidative degradation of the algal subcellular structures might be the cause of decreased resistance to fungal infection.

\section{Secondary metabolites mediated fenton reaction facilitates the fungal infection}

Metabolomic analysis was performed for identification of the small molecules causing the oxidative stresses. The SPIs were collected at different infection stages, i.e., 1, 3 and 5 day post inoculation of the fungus into the algal cell cultures. It was found that the degradation activity of SPI collected on Day (D) 5 was significantly higher than that on D1, suggesting that the concentration of the metabolites of target increased over 5 days. Based on this, 62 metabolites which showed over 2-fold increases on D5 than that on D1 were selected, most of which were organic acid, dipeptide, amino acid and derivatives. Ten metabolites, including tyramine, trimethoprim, indole-3-carboxylic acid, hordenine, deoxycytidine, 4pyridoxic acid, lumichrome, 3-hydroxyanthranilic acid (3-HAA), baclofen and cyclohexylamine, with the phenol/quinone/aromatic structure, were retrieved manually (Fig. 3), since such types of compounds are known to be able to mediate the fenton reaction producing hydroxyl peroxide (Arantes et al., 2012; Eastwood et al., 2011) . 
When these substances were added into the algal cell culture, it was firstly observed that hordenine and 3HAA significantly reduced the contents of carbohydrates while tyramine, hordenine and cyclohexylamine caused degradation of the pigments in the treated algal cells (Fig. 4A). Secondly, the potential infectionprompting effect of the candidate metabolites was checked. On the $3^{\text {rd }}$ dpi (day post infection), the algal cells pre-treated with either hordenine or 3-HAA showed significantly enhanced infection ratio than that of the control and other compounds (Fig. 4B).

The fenton reaction is initiated from the reduction of $\mathrm{Fe}^{3+}$ to $\mathrm{Fe}^{2+}$, which is the key factor for driving fenton reaction (Kameshwar \& Qin, 2018). Thus the reducing activities of the 10 candidate metabolites were tested. Among them, 3-HAA showed the strongest activity in reducing $\mathrm{Fe}^{3+}$ to $\mathrm{Fe}^{2+}$ at $4 \mathrm{~h}$ (Fig. $4 \mathrm{C}$ ). Additionally, 3-HAA and hordenine generated hydroxyl radical in the assay with DMSO as substrate (Fig. 4D). The intracellular concentration of hydrogen peroxide $\left(\mathrm{H}_{2} \mathrm{O}_{2}\right)$, an important intermediate of the fenton reaction, was measured after staining with the fluorescence DCFH-DA. The result showed that in the hordenine-treating algal cells the concentration of $\mathrm{H}_{2} \mathrm{O}_{2}$ continued increasing during $48 \mathrm{~h}$, while the level of $\mathrm{H}_{2} \mathrm{O}_{2}$ in the 3-HAA-treating algal cells transiently increased during $24 \mathrm{~h}$ and the gradually decreased (Fig. 4E).

These results taken together indicated 3-HAA and hordenine are the components in SPI which caused oxidative stresses in the algal cells through mediating fenton reaction. The metabolite 3-HAA is an intermediate of kynurenine pathway, and has been found in bacteria, yeast, fungi, plants and mammals and was reported to be a fungal producing mediator that was widely applied in fenton processes in dye decolorization due to its ability in reducing $\mathrm{Fe}^{3+}$ to $\mathrm{Fe}^{2+}$ (Santana \& Aguiar, 2015; Santana et al., 2019). It is also a generator of free radicals through its auto-oxidation (Breton et al., 2000; Li et al., 2001). Thus, the generation of $\mathrm{H}_{2} \mathrm{O}_{2}$ from the auto-oxidation of 3-HAA could not be excluded here. Hordenine, originally detected in barley and also isolated from marine algal Phyllophora nervosa (Guven et al., 2010; Mann et al., 1963), is a phenethylamine alkaloid with various bioactivities, including antibiotic activity against microorganisms, inhibition of quorum sensing and biofilm formation (Rao, 1970; Zhou et al., 2018). It was reported that hordenine was responsible for the protective responses of plants to various stresses through jasmonate dependent defense pathway (Ishiai et al., 2016), and also acted as an plant allelochemical that can inhibit the growth of weed or defend against pathogens attack (Kotzamani et al., 2021; Lebecque et al., 2018).

\section{Application of antioxidant to inhibit the fungal infection}

As the oxidative stresses caused by the SPI impaired the algal cell structures and promoted the fungal infection process, an exogenous antioxidant was introduced to relieving such oxidative stress in the culture to inhibit the infection. BHA is one of the most commonly used synthetic antioxidants in food and biodiesel fuels to prevent oxidation for its low cost, high stability and effectiveness (Rodil et al., 2012; Ryu, 2010; Sahraee et al., 2019; Xu et al., 2021). Additionally, BHA is biosafe and environmental-friendly, rendering its application in the aquaculture industry (Additives et al., 2018; Williams et al., 1999). BHA was added into the infection system at different final concentrations (i.e. 2 ppm, 7 ppm and 12 ppm) (Fig. 
5). The infection ratio of the newly infected algal cells was calculated to reflect the infection inhibitory effect. Compared to the untreated $H$. pluvialis culture, addition of BHA at 2 ppm delayed the complete fungal infection for 1 day. Elevating the concentration of BHA to $7 \mathrm{ppm}$ decreased the infection greatly, and the infection was only about $30 \%$ on day 5 , while the untreated group was $100 \%$ infected. Application of $12 \mathrm{ppm}$ BHA to the system completely suppressed the fungal infection.

According to all the results described above, a model was proposed herein to illustrate the major findings of this study. Production of secondary metabolites such as 3-HAA and hordenine in the infection system mediated the generation of hydroxyl radical via the fenton reaction, the most reactive free radicals among various ROS, which disrupt the subcellular components of the $H$. pluvialis cells and make the algal cells more susceptible to the infection. However, by adding $12 \mathrm{ppm}$ of the antioxidant BHA to the culture, the fungal infection was completely abolished, indicating the oxidative burst is essential for the pathogens to infest.

\section{Conclusion}

In this study, two secondary metabolites (i.e. 3-HAA and hordenine) were identified from the fungal infection system, which can drive fenton reaction thereby causing severe oxidative stresses on the host cells. Based on these findings, the antioxidant BHA was used to successfully block the fungal infection. This proof-of-concept study indicated utilization of antioxidants represents a novel strategy to reduce the fungal infection in microalgal mass culture.

\section{Methods}

\section{Strains and culturing conditions}

H. pluvialis cells were maintained in our laboratory (Lin et al., 2021) and cultured in the BG11 growth medium at $21-23^{\circ} \mathrm{C}$ under continuous illumination $\left(20 \mu \mathrm{mol} \cdot \mathrm{m}^{-2} \cdot \mathrm{s}^{-1}\right)$. The fungal parasite $P$. sedebokerense used in this study was isolated in the previous study (Lin et al., 2021). P. sedebokerense cells were grown in the fungal growth medium supplemented with yeast extract and peptone (Hoffman et al., 2008) on an orbital shaker at a speed of $150 \mathrm{rpm}$ maintained at $30^{\circ} \mathrm{C}$.

\section{Preparation of the supernatant post infection (SPI) and SPI incubation assay}

When the $\mathrm{OD}_{600}$ of the fungal cell cultures reached 0.03 within 5 days, $1 \%(v / v)$ of the fungal cells was inoculated in the algal cell cultures (about $3.0 \times 10^{5}$ algal cells $\mathrm{mL}^{-1}$ ). The mixture was then incubated on an orbital shaker at a speed of $150 \mathrm{rpm}$ at $30^{\circ} \mathrm{C}$. Samplings were performed on a daily basis and the infection ratios (Gutman et al., 2011) were determined by cell counting under microscope (Olympus, BX53 with a DP70 CCD camera). To prepare for the SPI, when the algal cells were completely infected by the parasitic fungus, the culture mixture was centrifuged at 2,100 $\mathrm{g}$ for $5 \mathrm{~min}$ to collect the supernatants and was filtrated by using double-layered $1.2 \mu \mathrm{m}$ glass microfiber filters (Whatman, UK), heated at $95^{\circ} \mathrm{C}$ in water bath for $15 \mathrm{~min}$ and were stored at $4^{\circ} \mathrm{C}$, such liquid was termed as supernatant post infection (SPI). 
For detecting the infection enhancing activity of the SPI, $100 \mathrm{~mL}$ of algal cells with density of $5.0 \times$ $10^{5}$ cells $\mathrm{mL}^{-1}$ from exponential growth phase was harvested at $700 \mathrm{~g}$ for $3 \mathrm{~min}$, the pellet was pre-treated with $100 \mathrm{~mL} \mathrm{SPI}$ or BG11 medium for $48 \mathrm{~h}$ at $30^{\circ} \mathrm{C}$, under light intensity of $20 \mu \mathrm{mol} \cdot \mathrm{m}^{-2} \cdot \mathrm{s}^{-1}$ at $150 \mathrm{rpm}$. The liquid was removed via centrifugation at $700 \mathrm{~g}$ for $3 \mathrm{~min}$, and the cell pellet was re-suspended in 100 $\mathrm{mL}$ of BG11 medium. Fungal cells were inoculated in the algal cell cultures, samples of each experiment were checked with microscope to count the prevalence of infection every day as described above. Three independent biological replicates were conducted for each sample and the quantitative data were presented as mean \pm S.D. $(n=3) . * *, p<0.01$ (Student's t-test).

\section{Transmission electron microscopy and starch staining}

Samples of healthy H. pluvialis cells that grown at logarithmic phase, or incubated with SPI for 3 days were centrifuged at $700 \mathrm{~g}$ for $3 \mathrm{~min}$, respectively. The cell pellet of each sample was washed twice with fresh BG11 medium, and was fixed overnight in $2.5 \%(\mathrm{w} / \mathrm{v})$ glutaraldehyde at $4^{\circ} \mathrm{C}$. The flowing sample processing procedures included osmium fixation, dehydration, infiltration, polymerization, section and staining (Wayama et al., 2013). The fixed and stained samples were examined with transmission electron microscope (FEl, Tecnai G2 20 TWIN, $0.24 \mathrm{~nm} / 200 \mathrm{kV}$ ). For starch staining, 2\% $(w / v)$ of the Lugol's solution was added to the algal cell samples and incubated for $5 \mathrm{~min}$. The starch granules were observed under light microscope.

\section{Biochemical analysis}

SPI or BG11 medium pretreated algal cells were pelleted via centrifuged at $700 \mathrm{~g}$ for 3 min and were washed with distilled $\mathrm{H}_{2} \mathrm{O}$. To extract the pigments, the cell pellets that grinded in mortar on ice under dim light were extracted with dimethyl sulfoxide (DMSO, for several times until the cell pellets turned colorless). The cellular contents of pigments chlorophyll and carotenoid were quantified by spectrophotometers. To analyze the content of remaining carbohydrates, the cells were hydrolyzed by 3 $\mathrm{M}$ trifluoroacetic acid (TFA) for $4 \mathrm{~h}$ at $98^{\circ} \mathrm{C}$, dried by nitrogen gas and re-established in distilled $\mathrm{H}_{2} \mathrm{O}$. After filtrated with $0.22 \mu \mathrm{m}$ micro-aperture filter membrane (Merck Millipore, USA), the sugar content in the sample was analyzed by an ICS 5000+ Ion Chromatography (IC) system (Thermo-Fisher Scientific, USA). Thiobarburic acid (TBA) assay was used for detecting oxidative activity of SPI. Fenton reagents $(0.83$ $\mathrm{mM}$ ferrous ions and $30 \mathrm{mM}$ hydrogen peroxide) were used as the positive control. The Malondialdehyde (MDA) kit (Jiancheng Bioengineering Institute, Nanjing, China) was introduced to measure the in vivo lipid peroxidation in the SPI treated $H$. pluvialis cells. Ferrozine assay was conducted to measure the ferric ions reducing activity (Gibbs, 1976).

\section{Analysis of the hydroxyl radical and hydrogen peroxide production}

The hydroxyl radicals oxidize dimethyl sulfoxide (DMSO) and generate formaldehyde, which can react with 2, 4-dinitrophenylhydrazine (DNPH) and form hydrazone (HCHO-DNPH). HCHO-DNPH can be analyzed by HPLC, eluted peaks of HCHO-DNPH with maximum absorbance at $355 \mathrm{~nm}$ was 
targeted (Tai et al., 2004). Fenton reagent containing $0.2 \mathrm{mM} \mathrm{Fe}^{2+}$ and $8 \mathrm{mM} \mathrm{H}_{2} \mathrm{O}_{2}$ was used as the positive control to generate hydroxyl radicals. To measure the intracellular hydrogen peroxide $\left(\mathrm{H}_{2} \mathrm{O}_{2}\right)$, the fluorescence dye 2', 7'-dichlorodihydrofluorescein diacetate (DCFH-DA, Sigma, USA) was introduced to stain the treated algal cells (Gwak et al., 2014). The stained cells were analyzed by using the flow cytometer and the fluorescence intensity (excitation wavelength at $488 \mathrm{~nm}$ ) of the cells reflected the intracellular $\mathrm{H}_{2} \mathrm{O}_{2}$ level.

\section{Metabolomics}

The SPI on day 1, 3 and 5 were collected respectively, and the supernatants were used for the ultra-high performance liquid chromatography coupled with hybrid quadrupole time-of-flight mass spectrometry (UHPLC-QTOF-MS) analysis. Two independent biological repeats were conducted for each sample. Fold change (peak intensity of D5/D1) > 3 was screened and compounds with phenol/quinone/aromatic moiety (Korripally et al., 2013) were particularly targeted. Target reagents were purchased from SigmaAldrich, cyclohexylamine was dissolved as $2 \%(v / v)$ solution in BG11 medium and the other 9 reagents were dissolved as $0.2 \%(\mathrm{w} / \mathrm{v})$ solution in BG11 medium. These solutions were put in a $95^{\circ} \mathrm{C}$ water bath for $15 \mathrm{~min}$ and cooled down and applied appropriate ultrasonic treatment to enhance the dissolution, the supernatants of each solutions were collected to further test their activity.

\section{RNA extraction and RNA-seq analysis}

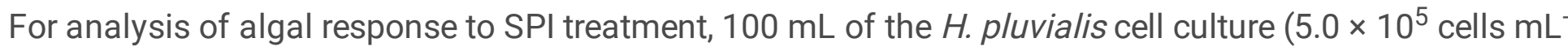

${ }^{1}$ ) were incubated in SPI or BG11 medium (as control), respectively for $24 \mathrm{~h}$. One $\mathrm{mL}$ of the algal cell cultures was then centrifuged to collect the pellet, and was frozen with liquid nitrogen immediately. The RNA extraction and RNA-seq analysis were performed according to the methods described by Ma et al. (Ma et al., 2020). Differentially expressed (DE) genes with $\log _{2} F C>1.5$ was considered, and the upand down- regulated gens was checked and analyzed manually. Three independent biological repeats were conducted for each sample.

\section{Declarations}

\section{Credit authorship contribution statement}

Hailong Yan: Data curation and Writing - original draft. Haiyan Ma: Data curation and Writing - original draft. Yanhua Li: Methodology. Liang Zhao: Data curation and Software. Juan Lin: Resources. Qikun Jia: Data curation. Danxiang Han and Qiang Hu: Funding acquisition and Writing - review.

\section{Acknowledgments}

This work was supported by the National Natural Science Foundation of China $(31570304 ; 31700359)$ and National Science Foundation of Hubei Province (2015CFA022). Wuhan Branch, Supercomputing Center (Chinese Academy of Sciences, China) provided support on the transcriptomic analysis. BioTree 
(Biotechnology Co., Ltd. Shanghai, China) provided the support on metabolomics analysis. We thank Engineer Zhenfei Xin (Institute of Hydrobiology, Chinese Academy of Sciences, Wuhan, China) for assistance in electron microscopy and Dr. Hu Jin (Center for Microalgal Biotechnology and Biofuels, Institute of Hydrobiology, Chinese Academy of Sciences) for offering kind advice in submitting the manuscript.

\section{Data availability}

Transcriptome data is available at NCBI Sequence Read Archive (https://www.ncbi.nlm.nih.gov/sra) with accession number PRJNA720251.

\section{Declaration of Competing Interest}

The authors declare that they have no competing financial interests or personal relationships that could have appeared to influence the work reported in this paper.

\section{References}

1. Additives, E.Panel o., Feed, P.o.S.u.i.A., Rychen, G., Aquilina, G., Azimonti, G., Bampidis, V., Bastos, M.d.L., Bories, G., Chesson, A., Cocconcelli, P.S., Flachowsky, G., Kolar, B., Kouba, M., López-Alonso, M., Puente, S.L., Mantovani, A., Mayo, B., Ramos, F., Saarela, M., Villa, R.E., Wallace, R.J., Wester, P., Lundebye, A.-K., Nebbia, C., Renshaw, D., Innocenti, M.L., Gropp, J. 2018. Safety and efficacy of butylated hydroxyanisole (BHA) as a feed additive for all animal species. EFSA Journal, 16(3), e05215.

2. Arantes, V., Jellison, J., Goodell, B. 2012. Peculiarities of brown-rot fungi and biochemical Fenton reaction with regard to their potential as a model for bioprocessing biomass. Applied Microbiology and Biotechnology, 94(2), 323-338.

3. Asatryan, A., Boussiba, S., Zarka, A. 2019. Stimulation and Isolation of Paraphysoderma sedebokerense (Blastocladiomycota) Propagules and Their Infection Capacity Toward Their Host Under Different Physiological and Environmental Conditions. Frontiers in Cellular and Infection Microbiology, 9, 72.

4. Borowitzka, M.A., Vonshak, A. 2017. Scaling up microalgal cultures to commercial scale. European Journal of Phycology, 52(4), 407-418.

5. Breton, J., Avanzi, N., Magagnin, S., Covini, N., Magistrelli, G., Cozzi, L., Isacchi, A. 2000. Functional characterization and mechanism of action of recombinant human kynurenine 3-hydroxylase. European Journal of Biochemistry, 267(4), 1092-1099.

6. Carney, L.T., Lane, T.W. 2014. Parasites in algae mass culture. Frontiers in Microbiology, 5, 278.

7. Choi, H.I., Hwang, S.-W., Sim, S.J. 2019. Comprehensive approach to improving life-cycle $\mathrm{CO} 2$ reduction efficiency of microalgal biorefineries: A review. Bioresource Technology, 291, 121879. 
8. Damiani, M.C., Popovich, C.A., Constenla, D., Leonardi, P.I. 2010. Lipid analysis in Haematococcus pluvialis to assess its potential use as a biodiesel feedstock. Bioresource Technology, 101(11), 3801-3807.

9. Ding, Y., Zhang, A., Wen, X., Wang, Z., Wang, K., Geng, Y., Li, Y. 2020. Application of surfactants for controlling destructive fungus contamination in mass cultivation of Haematococcus pluvialis. Bioresource Technology, 317, 124025.

10. Eastwood, D.C., Floudas, D., Binder, M., Majcherczyk, A., Schneider, P., Aerts, A., Asiegbu, F.O., Baker, S.E., Barry, K., Bendiksby, M., Blumentritt, M., Coutinho, P.M., Cullen, D., de Vries, R.P., Gathman, A., Goodell, B., Henrissat, B., Ihrmark, K., Kauserud, H., Kohler, A., LaButti, K., Lapidus, A., Lavin, J.L., Lee, Y.-H., Lindquist, E., Lilly, W., Lucas, S., Morin, E., Murat, C., Oguiza, J.A., Park, J., Pisabarro, A.G., Riley, R., Rosling, A., Salamov, A., Schmidt, O., Schmutz, J., Skrede, I., Stenlid, J., Wiebenga, A., Xie, X., Kües, U., Hibbett, D.S., Hoffmeister, D., Högberg, N., Martin, F., Grigoriev, I.V., Watkinson, S.C. 2011. The Plant Cell Wall-Decomposing Machinery Underlies the Functional Diversity of Forest Fungi. Science, 333(6043), 762-765.

11. Gibbs, C.R. 1976. Characterization and Application of Ferrozine Iron Reagent as a Ferrous Iron Indicator. Analytical Chemistry, 48(8), 1197-1201.

12. Gutman, J., Zarka, A., Boussiba, S. 2011. Evidence for the involvement of surface carbohydrates in the recognition of Haematococcus p/uvialis by the parasitic blastoclad Paraphysoderma sedebokerensis. Fungal Biology, 115(8), 803-811.

13. Gutman, J., Zarka, A., Boussiba, S. 2009. The host-range of Paraphysoderma sedebokerensis, a chytrid that infects Haematococcus pluvialis. European Journal of Phycology, 44(4), 509-514.

14. Guven, K.C., Percot, A., Sezik, E. 2010. Alkaloids in Marine Algae. Marine Drugs, 8(2), 269-284.

15. Gwak, Y., Hwang, Y.S., Wang, B.B., Kim, M., Jeong, J., Lee, C.G., Hu, Q., Han, D.X., Jin, E. 2014. Comparative analyses of lipidomes and transcriptomes reveal a concerted action of multiple defensive systems against photooxidative stress in Haematococcus pluvialis. Journal of Experimental Botany, 65(15), 4317-4334.

16. Han, D.X., Li, Y.T., Hu, Q. 2013. Astaxanthin in microalgae: pathways, functions and biotechnological implications. Algae, 28(2), 131-147.

17. Harker, M., Tsavalos, A.J., Young, A.J. 1996. Factors responsible for astaxanthin formation in the Chlorophyte Haematococcus pluvialis. Bioresource Technology, 55(3), 207-214.

18. Hasanuzzaman, M., Bhuyan, M.H.M.B., Zulfiqar, F., Raza, A., Mohsin, S.M., Al Mahmud, J., Fujita, M., Fotopoulos, V. 2020. Reactive Oxygen Species and Antioxidant Defense in Plants under Abiotic Stress: Revisiting the Crucial Role of a Universal Defense Regulator. Antioxidants, 9(8), 681.

19. Hoffman, Y., Aflalo, C., Zarka, A., Gutman, J., James, T.Y., Boussiba, S. 2008. Isolation and characterization of a novel chytrid species (phylum Blastocladiomycota), parasitic on the green alga Haematococcus. Mycological Research, 112(1), 70-81.

20. Hwang, S.-W., Choi, H.I., Sim, S.J. 2019. Acidic cultivation of Haematococcus pluvialis for improved astaxanthin production in the presence of a lethal fungus. Bioresource Technology, 278, 138-144. 
21. Ishiai, S., Kondo, H., Hattori, T., Mikami, M., Aoki, Y., Enoki, S., Suzuki, S. 2016. Hordenine is responsible for plant defense response through jasmonate-dependent defense pathway. Physiological and Molecular Plant Pathology, 96, 94-100.

22. Kameshwar, A.K.S., Qin, W.S. 2018. Molecular Networks of Postia placenta Involved in Degradation of Lignocellulosic Biomass Revealed from Metadata Analysis of Open Access Gene Expression Data. International Journal Of Biological Sciences, 14(3), 237-252.

23. Khoo, K.S., Lee, S.Y., Ooi, C.W., Fu, X., Miao, X., Ling, T.C., Show, P.L. 2019. Recent advances in biorefinery of astaxanthin from Haematococcus pluvialis. Bioresource Technology, 288, 121606.

24. Korripally, P., Timokhin, V.I., Houtman, C.J., Mozuch, M.D., Hammel, K.E. 2013. Evidence from Serpula lacrymans that 2,5-Dimethoxyhydroquinone Is a Lignocellulolytic Agent of Divergent Brown Rot Basidiomycetes. Applied and Environmental Microbiology, 79(7), 2377-2383.

25. Kotzamani, A., Vasilakoglou, I., Dhima, K., Moulas, A.N., Vaiou, M., Stefanou, S. 2021. Impact of Soil Salinity on Barley Allelopathic Potential and Main Secondary Metabolites Gramine and Hordenine. Journal of Plant Growth Regulation, 40(1), 137-146.

26. Lebecque, S., Crowet, J.M., Lins, L., Delory, B.M., du Jardin, P., Fauconnier, M.L., Deleu, M. 2018. Interaction between the barley allelochemical compounds gramine and hordenine and artificial lipid bilayers mimicking the plant plasma membrane. Scientific Reports, 8, 9784.

27. Li, K.C., Horanyi, P.S., Collins, R., Phillips, R.S., Eriksson, K.E.L. 2001. Investigation of the role of 3hydroxyanthranilic acid in the degradation of lignin by white-rot fungus Pycnoporus cinnabarinus. Enzyme and Microbial Technology, 28(4-5), 301-307.

28. Lin, J., Yan, H., Zhao, L., Li, Y., Nahidian, B., Zhu, M., Hu, Q., Han, D. 2021. Interaction between the cell walls of microalgal host and fungal carbohydrate-activate enzymes is essential for the pathogenic parasitism process. Environmental Microbiology, 23(9), 5114-5130.

29. Ma, H., Wu, X., Wei, Z., Zhao, L., Li, Z., Liang, Q., Zheng, J., Wang, Y., Li, Y., Huang, L., Hu, Q., Han, D. 2020. Functional divergence of diacylglycerol acyltransferases in the unicellular green alga Haematococcus pluvialis. Journal of Experimental Botany, 72(2), 510-524.

30. Maity, J.P., Bundschuh, J., Chen, C.-Y., Bhattacharya, P. 2014. Microalgae for third generation biofuel production, mitigation of greenhouse gas emissions and wastewater treatment: Present and future perspectives - A mini review. Energy, 78, 104-113.

31. Mann, J.D., Mudd, S.H., Steinhart, C.E. 1963. Alkaloids and Plant Metabolism.5. Distribution and Formation of Tyramine Methylpherase during Germination of Barley. Journal of Biological Chemistry, 238(2), 676-681.

32. Rao, G.S. 1970. Identity of Peyocactin, an Antibiotic from Peyote (Lophophora-Williamsii), and Hordenine. Journal of Pharmacy and Pharmacology, 22(7), 544-545.

33. Ren, Y., Deng, J., Huang, J., Wu, Z., Yi, L., Bi, Y., Chen, F. 2021. Using green alga Haematococcus pluvialis for astaxanthin and lipid co-production: Advances and outlook. Bioresource Technology, 340, 125736. 
34. Rodil, R., Quintana, J.B., Cela, R. 2012. Oxidation of synthetic phenolic antioxidants during water chlorination. Journal of Hazardous Materials, 199-200, 73-81.

35. Ryu, K. 2010. The characteristics of performance and exhaust emissions of a diesel engine using a biodiesel with antioxidants. Bioresource Technology, 101(1), S78-S82.

36. Sahraee, S., Milani, J.M., Regenstein, J.M., Kafil, H.S. 2019. Protection of foods against oxidative deterioration using edible films and coatings: A review. Food Bioscience, 32, 100451.

37. Santana, C.S., Aguiar, A. 2015. Effect of biological mediator, 3-hydroxyanthranilic acid, in dye decolorization by Fenton processes. International Biodeterioration \& Biodegradation, 104, 1-7.

38. Santana, C.S., Ramos, M.D.N., Velloso, C.C.V., Aguiar, A. 2019. Kinetic Evaluation of Dye Decolorization by Fenton Processes in the Presence of 3-Hydroxyanthranilic Acid. International Journal of Environmental Research and Public Health, 16(9), 1602.

39. Shah, M.M.R., Liang, Y., Cheng, J.J., Daroch, M. 2016. Astaxanthin-Producing Green Microalga Haematococcus pluvialis: From Single Cell to High Value Commercial Products. Frontiers in Plant Science, 7, 531.

40. Specht, E., Miyake-Stoner, S., Mayfield, S. 2010. Micro-algae come of age as a platform for recombinant protein production. Biotechnology Letters, 32(10), 1373-1383.

41. Strittmatter, M., Guerra, T., Silva, J., Gachon, C.M.M. 2016. A new flagellated dispersion stage in Paraphysoderma sedebokerense, a pathogen of Haematococcus pluvialis. Journal of Applied Phycology, 28(3), 1553-1558.

42. Tai, C., Peng, J.F., Liu, J.F., Jiang, G.B., Zou, H. 2004. Determination of hydroxyl radicals in advanced oxidation processes with dimethyl sulfoxide trapping and liquid chromatography. Analytica Chimica Acta, 527(1), 73-80.

43. Torres-Tiji, Y., Fields, F.J., Mayfield, S.P. 2020. Microalgae as a future food source. Biotechnology Advances, 41, 107536.

44. Torres, M.A., Jones, J.D.G., Dangl, J.L. 2006. Reactive oxygen species signaling in response to pathogens. Plant Physiology, 141(2), 373-378.

45. Wayama, M., Ota, S., Matsuura, H., Nango, N., Hirata, A., Kawano, S. 2013. Three-Dimensional Ultrastructural Study of Oil and Astaxanthin Accumulation during Encystment in the Green Alga Haematococcus pluvialis. PLOS ONE, 8(1), e53618.

46. Williams, G.M., latropoulos, M.J., Whysner, J. 1999. Safety Assessment of Butylated Hydroxyanisole and Butylated Hydroxytoluene as Antioxidant Food Additives. Food and Chemical Toxicology, 37(9), 1027-1038.

47. Xu, X., Liu, A., Hu, S., Ares, I., Martínez-Larrañaga, M.-R., Wang, X., Martínez, M., Anadón, A., Martínez, M.-A. 2021. Synthetic phenolic antioxidants: Metabolism, hazards and mechanism of action. Food Chemistry, 353, 129488.

48. Zhou, J.W., Luo, H.Z., Jiang, H., Jian, T.K., Chen, Z.Q., Jia, A.Q. 2018. Hordenine: A Novel Quorum Sensing Inhibitor and Antibiofilm Agent against Pseudomonas aeruginosa. Journal of Agricultural and Food Chemistry, 66(7), 1620-1628. 

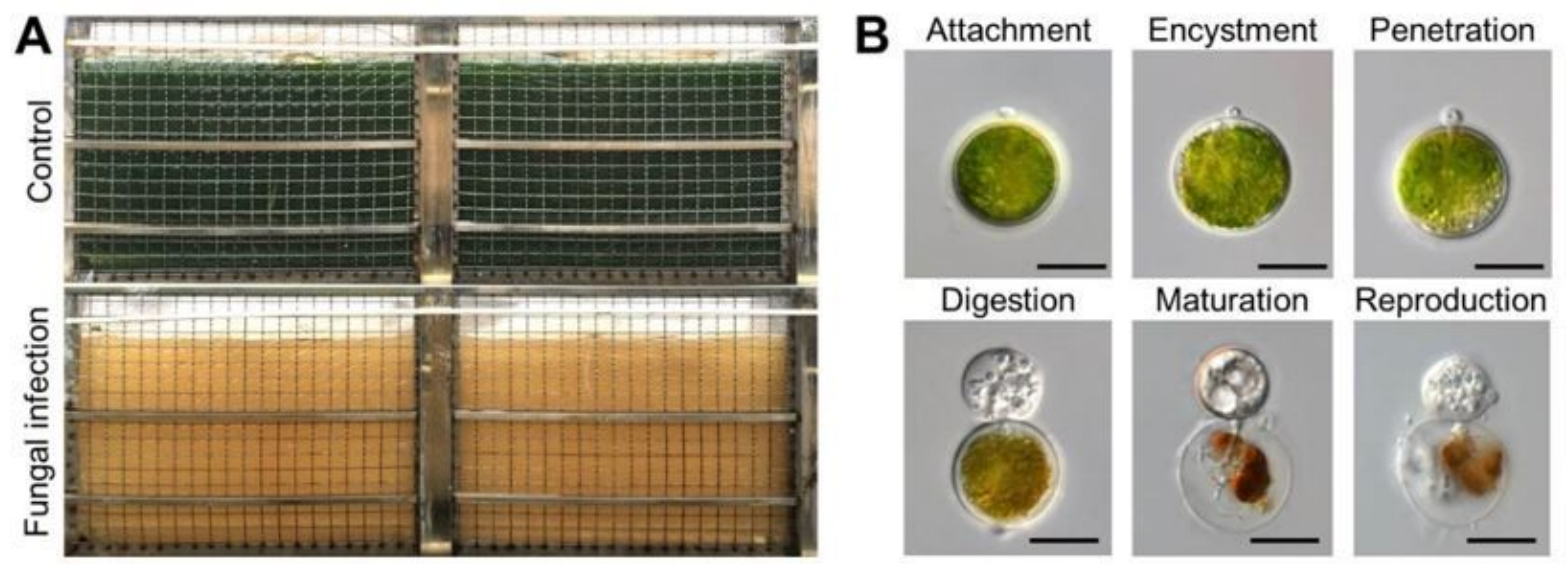

Assimilation



$3^{\text {rd }}$ Day Post Infection
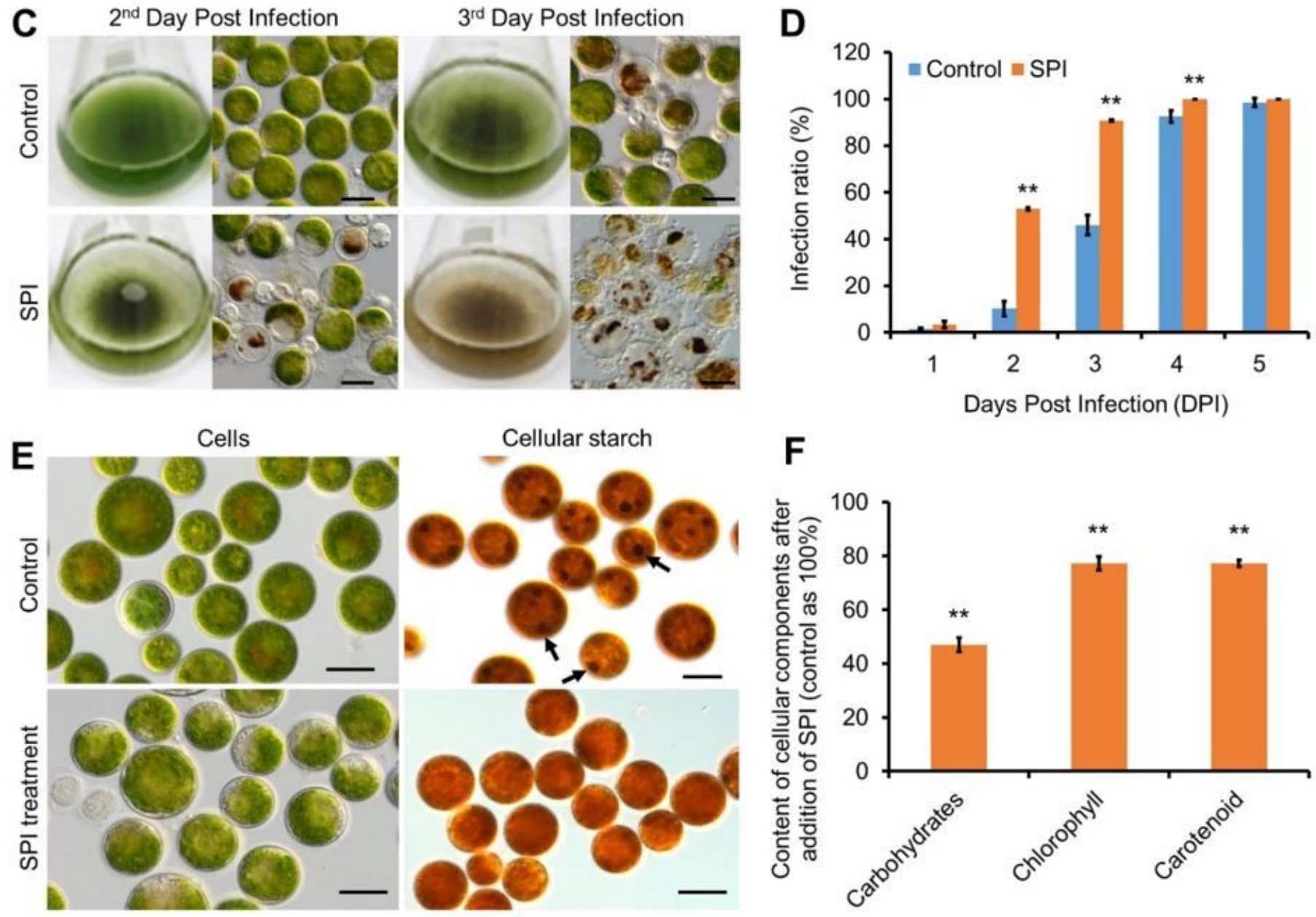

$\mathbf{F}$



Figure 1

Fungal contamination crushed the growth of Haematococcus pluvialis culture and the supernatant post infection (SPI) enhanced the fungal infection process. (A-B) Crush of $\mathrm{H}$. pluvialis algal culture after contaminated by fungal parasite P. sedebokerense. (C-D) SPI enhanced the fungal infection process. (E-F) Decomposing effects of SPI on the algal cell. Bars $=20 \mu \mathrm{m}$. 
A
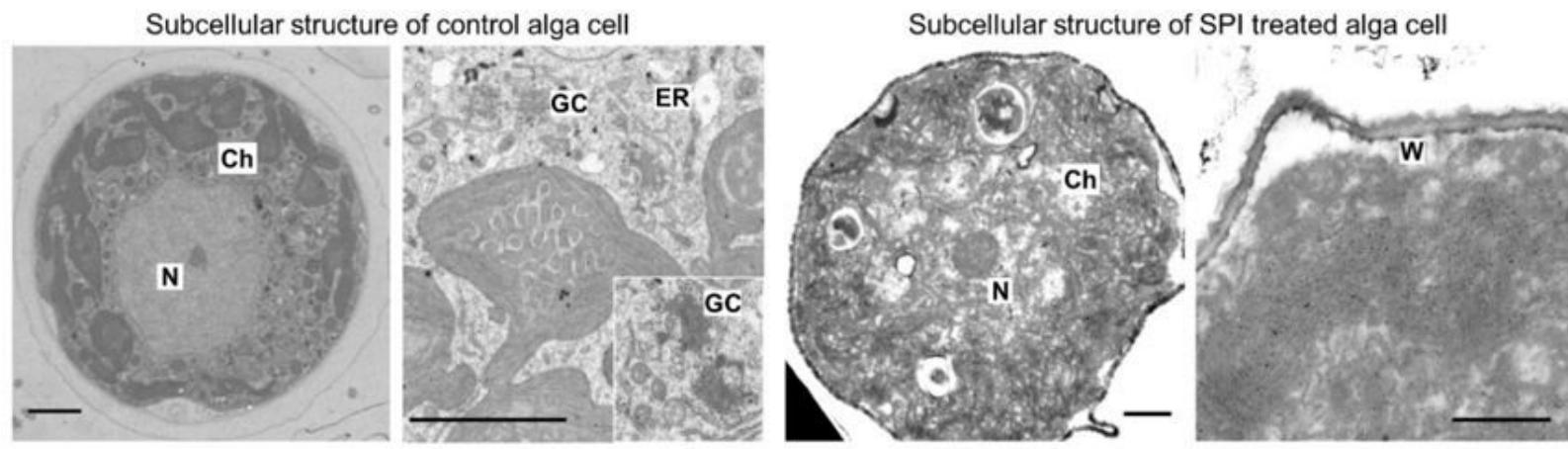

B

B Glutathione peroxidase

Glutathione reductase

Glutathione reductase, chloroplastic

Glutathione S-transferase

Phosphatidylinositol transfer protein CSR1

Probable L-gulonolactone oxidase 6

Purple acid phosphatase 17

Putative D-arabinono-1,4-lactone oxidase

Lipoxygenase homology domain-containing protein 1

3-ketoacyl-CoA synthase 1

3-ketoacyl-CoA synthase 4

3-ketoacyl-CoA synthase 5

Alpha/beta-hydrolase

Gastric triacylglycerol lipase Lipase/esterase

Lysosomal acid lipase/cholesteryl ester hydrolase-like

Putative acyl-CoA-binding protein

S-acyl fatty acid synthase thioesterase, medium chain

Amino-acid permease BAT1 homolog

Ammonium transporter 1 member 1

Ammonium transporter 1 member 2

Putative ammonium transporter 1 member 5

Urea-proton symporter DUR3

Glutamate synthase [NADH], amyloplastic

Glutamate synthase 1 [NADH], chloroplastic

Glutamate synthase 2 [NADH], chloroplastic

Glutamine synthetase cytosolic isozyme

Glutamine synthetase, chloroplastic

Hydroxyproline-rich glycoprotein, stress-induced

Hypersensitive-induced response protein 1

Hypersensitive-induced response protein 3


E



\section{Figure 2}

The SPI caused alga structure degradation and exhibited oxidative activities both in vivo and in vitro. (A) TEM observation on the algal cells treated with SPI. N, nuclear. W, cell wall. Ch, chloroplast. GC, Golgi complex. ER, endoplasmic reticulum. (B) RNA-seq analysis. (C) Oxidative activities of the SPI. (D) Lipid peroxidation in host algal cells treated with the SPI. (E) Hydroxyl radical detection. Bars $=2 \mu \mathrm{m}$. 


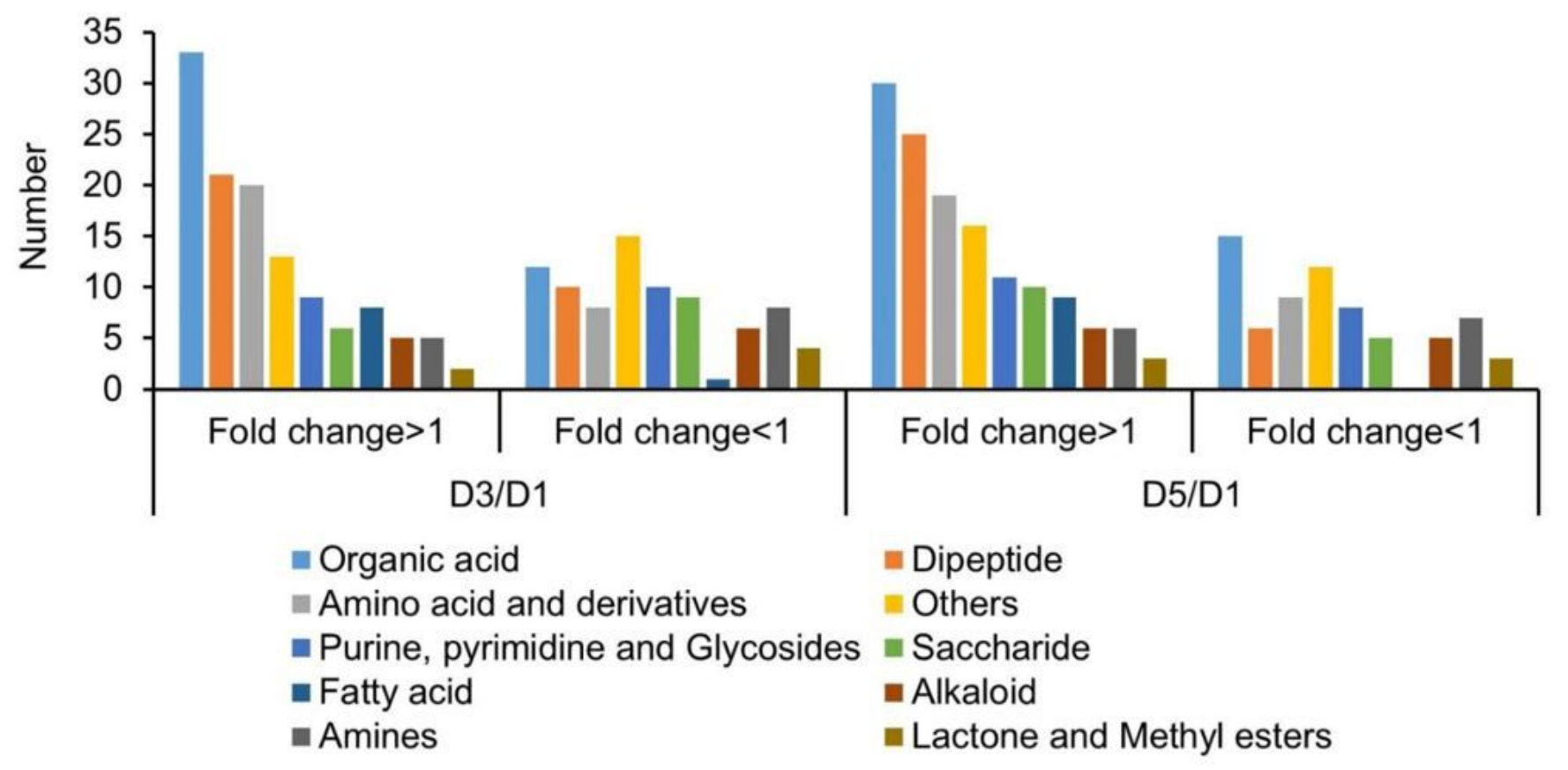<smiles>NCCc1ccc(O)cc1</smiles>

Tyramine 1: 22.8: 32<smiles>NC1CCCCC1</smiles>

Cyclohexylamine 1: $1.6: 4.5$<smiles>COc1cc(Cc2cc(N)n(N)n2)cc(O)c1O</smiles>

Trimethoprim 1: 13.8: 25.1

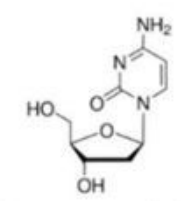

Deoxycytidine 1: $0.9: 4.1$<smiles></smiles>

Indole-3-carboxylic acid

1: $1.4: 11$<smiles>Cc1ncc(CO)c(O)c1O</smiles>

4-Pyridoxic acid

1: $1.7: 3.6$<smiles>CN(C)CCc1ccc(O)cc1</smiles>

Hordenine 1: $5: 9.3$

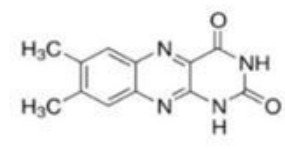

Lumichrome 1: $0.7: 3.6$<smiles>NCC(CO)(CC(=O)O)Cc1ccc(Cl)cc1</smiles>

Baclofen 1: $4.5: 8.1$<smiles>O=C(O)C1CCCC(O)C1</smiles>

3-HAA 1: $3.3: 3.1$

\section{Figure 3}

Identification of the metabolites with putative functions in causing oxidative stresses in the host algal cells through comparative metabolomics analysis. The formula of each metabolites was referred to Sigma-Aldrich and KEGG data-base (https://www.genome.jp/kegg/), the relative content of each metabolites from D1: D3: D5 was also indicated below. 

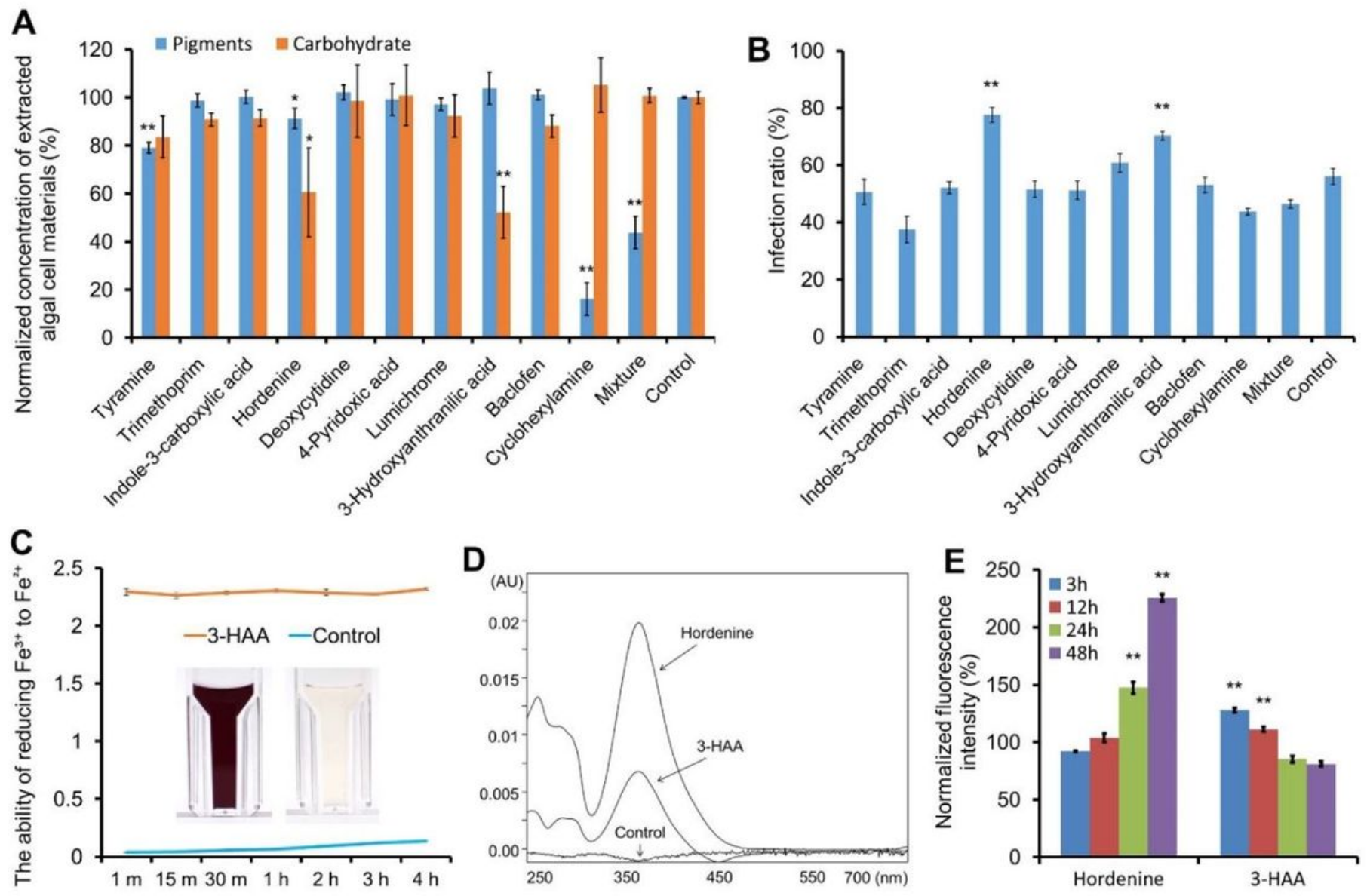

Figure 4

Effects of the screened metabolites on the algal cellular composition and infection process. (A-B) Activity verification of the screened metabolites. (C) 3-HAA reduced Fe3+ to Fe2+. (D) Hydroxyl radical production. (E) H2O2 level inside cells. 

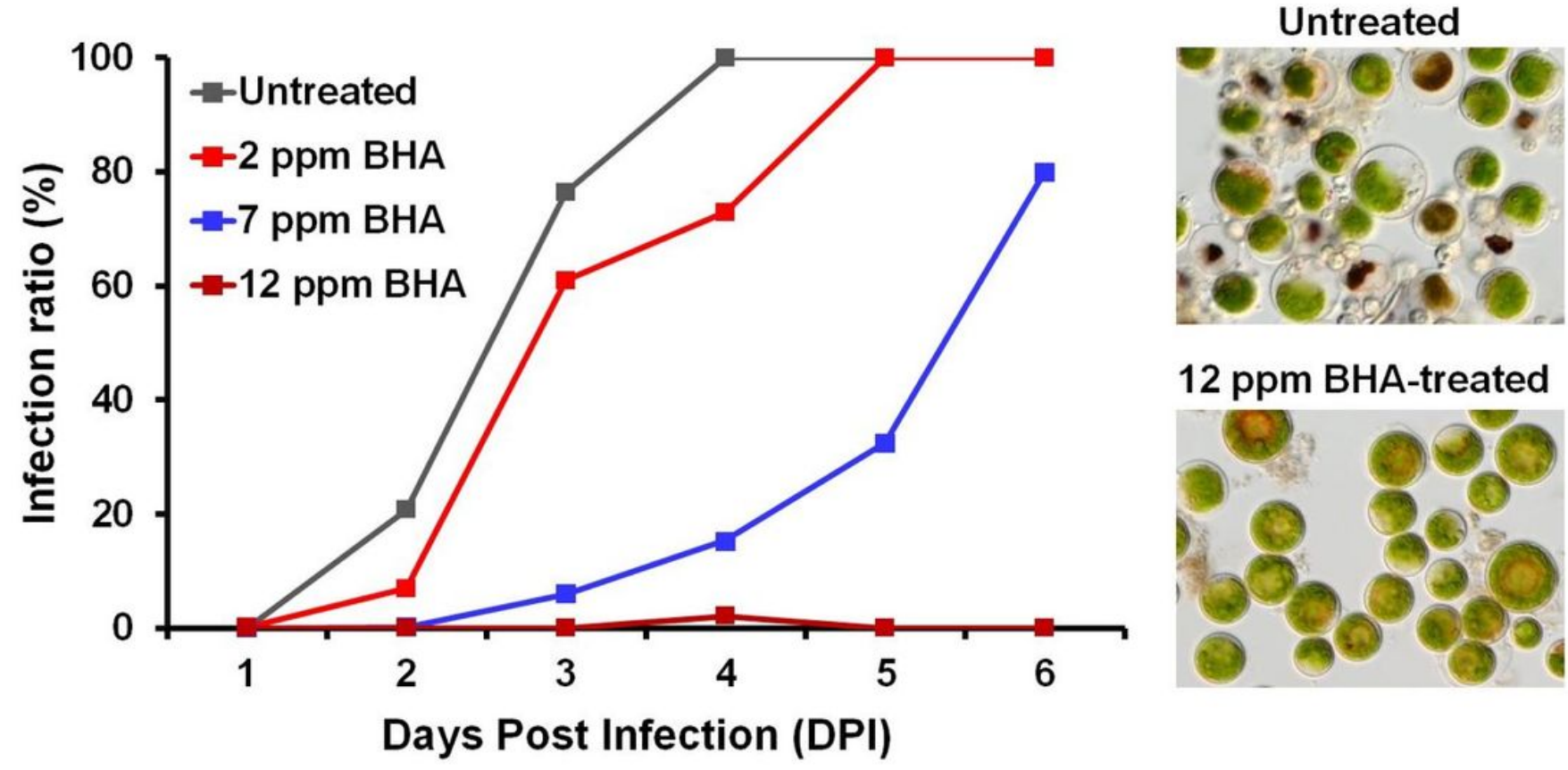

12 ppm BHA-treated

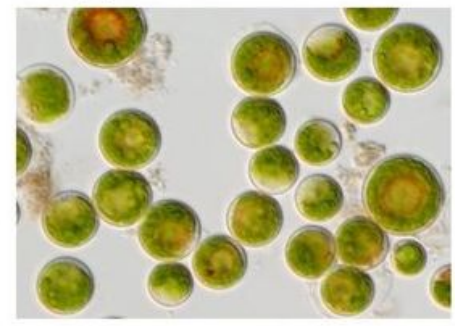

Figure 5

The inhibition effect of BHA to the fungal infection at different final concentrations. 